Portland State University

PDXScholar

$12-28-2014$

\title{
Force Chains as the Link Between Particle and Bulk Friction Angles in Granular Material
}

\author{
Adam M. Booth \\ Portland State University, boothad@pdx.edu \\ Ryan Hurley \\ California Institute of Technology \\ Michael P. Lamb \\ California Institute of Technology \\ José E. Andrade \\ California Institute of Technology
}

Follow this and additional works at: https://pdxscholar.library.pdx.edu/geology_fac

Part of the Geology Commons

Let us know how access to this document benefits you.

\section{Citation Details}

Booth, A. M., Hurley, R., Lamb, M. P., \& Andrade, J. E. (2014). Force chains as the link between particle and bulk friction angles in granular material. Geophysical Research Letters.

This Article is brought to you for free and open access. It has been accepted for inclusion in Geology Faculty Publications and Presentations by an authorized administrator of PDXScholar. Please contact us if we can make this document more accessible: pdxscholar@pdx.edu. 


\section{Geophysical Research Letters}

\section{RESEARCH LETTER \\ 10.1002/2014GL061981 \\ Key Points: \\ - Documents a continuous transition between grain-pocket and bulk friction angles \\ - Important for patchy sediment in fluvial, aeolian, and gravitational transport \\ - Bridges gap between macroscale constitutive laws and particle-based laws}

Supporting Information:

- Table S1

Correspondence to:

A. M. Booth,

boothad@pdx.edu

Citation:

Booth, A. M., R. Hurley, M. P. Lamb, and J. E. Andrade (2014), Force chains as the link between particle and bulk friction angles in granular material, Geophys. Res. Lett., 41, 8862-8869, doi:10.1002/ 2014 GL061981.

Received 22 SEP 2014 Accepted 1 DEC 2014 Accepted article online 4 DEC 2014 Published online 18 DEC 2014

\section{Force chains as the link between particle and bulk friction angles in granular material}

\author{
Adam M. Booth ${ }^{1,2}$, Ryan Hurley ${ }^{3}$, Michael P. Lamb², and José E. Andrade ${ }^{3}$ \\ ${ }^{1}$ Department of Geology, Portland State University, Portland, Oregon, USA, ${ }^{2}$ Division of Geological and Planetary Sciences, \\ California Institute of Technology, Pasadena, California, USA, ${ }^{3}$ Division of Engineering and Applied Science, Department of \\ Mechanical and Civil Engineering, California Institute of Technology, Pasadena, California, USA
}

Abstract From sediment transport in rivers to landslides, predictions of granular motion rely on a Mohr-Coulomb failure criterion parameterized by a friction angle. Measured friction angles are generally large for single grains, smaller for large numbers of grains, and no theory exists for intermediate numbers of grains. We propose that a continuum of friction angles exists between single-grain and bulk friction angles due to grain-to-grain force chains. Physical experiments, probabilistic modeling, and discrete element modeling demonstrate that friction angles decrease by up to $15^{\circ}$ as the number of potentially mobile grains increases from 1 to $\sim 20$. Decreased stability occurs as longer force chains more effectively dislodge downslope "keystone" grains, implying that bulk friction angles are set by the statistics of single-grain friction angles. Both angles are distinct from and generally larger than grain contact-point friction, with implications for a variety of sediment transport processes involving small clusters of grains.

\section{Introduction}

Predicting the movement of granular materials in geological settings often relies on a Mohr-Coulomb failure criterion where resistance to motion is determined by the product of pressure normal to the failure surface and the tangent of a friction angle accounting for the surface's roughness:

$$
\tau_{r}=\sigma_{n} \tan \varphi,
$$

where $\tau_{r}$ is resisting shear stress, $\sigma_{n}$ is normal stress, and $\varphi$ is friction angle. The friction angle in equation (1) is empirical and has different interpretations based on the specific granular transport process. For example, transport of a single grain in a fluvial system is a function of the grain-pocket friction angle, defined as the bed inclination angle at which that particle would roll or slide out of its specific location on the bed [Wiberg and Smith, 1987]. The grain-pocket friction angle is distinct from the friction angle describing slip at grain-to-grain contacts (i.e., contact-point friction), which is a material property set by roughness at the subgrain scale and unaffected by grain size, shape, or pocket geometry. The grain-pocket friction angle is likely different than the contact-point friction angle because grains can have multiple contact points, and destabilization can occur by rolling in addition to slip at the contact point. Measurements of grain-pocket angles are quite variable and range from $\sim 20^{\circ}$ to $>90^{\circ}$ for mixed grain sizes [Miller and Byrne, 1966; Kirchner et al., 1990; Buffington et al., 1992]. In contrast to grain-pocket friction angles, slope stability analysis in soil mechanics requires the angle of internal friction for the bulk material, defined as the slope of a Mohr-Coulomb failure envelope constructed from laboratory tests on samples containing large numbers of grains [Lambe and Whitman, 1969]. Bulk friction angles of cohesionless materials are $~ 30^{\circ}$ to $50^{\circ}$ and much less variable than individual grain-pocket friction angles [Selby, 1993]. Numerous studies have independently addressed the end-member cases of single-grain and bulk friction angles, but few have investigated how these angles might be related, or the friction angles for intermediate numbers of grains between these end-member states.

Many naturally occurring sediment transport processes involve assemblies of small numbers of grains where neither a grain-pocket nor bulk friction angle is appropriate. Wind ripples can have steep lee faces with lengths of several tens of grain diameters, especially coarse-grained granule ripples [Sharp, 1963]. In steep bedrock landscapes, small piles of loose grains trapped upslope of vegetation or other roughness obstacles are systematically larger and steeper where particle size approaches obstacle size [DiBiase et al., 2012; DiBiase and Lamb, 2013; Lamb et al., 2013]. Talus accumulations with dimensions approaching the size of their constituent blocks are also common, and their slopes tend to steepen near the top [Behre, 1933], suggesting a 
transition from bulk friction angles lower on the pile to higher friction angles at the top. Mass failures and flows including rock avalanches, dry ravel, shallow landslides, riverbed failure, and debris flows can have thicknesses that are less than a few tens of grains [e.g., Takahashi, 2014]. In fluvial systems, bedload sediment often clusters into small patches or chains of similarly sized particles whose motion or stability is determined by force chains acting through grain contacts [Frey and Church, 2009, 2011; Scheingross et al., 2013]. As grains move along the bed they tend to become trapped or jammed behind particularly immobile grains creating clusters [Brayshaw et al., 1983], stone cells [Church et al., 1998], or step-pools [Church and Zimmermann, 2007] that require higher shear stresses to reinitiate movement [e.g., Estep and Dufek, 2012].

A limited number of previous experiments have identified a decrease in the friction angle of granular material as the number of displaced grains increases. In tilting box experiments with crushed stone, Carson [1977] observed that the gravel's maximum angle of stability was highest for the smallest avalanches and was lowest and consistent with the maximum angle of piles of the same material observed in the field for the largest avalanches. Bulk friction angles of coarse granular materials measured with direct shear or triaxial tests are often 5 to $15^{\circ}$ higher than the maximum surface slope of a pile of many grains when the apparatus size is less than $\sim 10$ grain diameters [e.g., Chandler, 1973; Kirkby and Statham, 1975; Carson, 1977]. Piles of granular material on platforms also attain progressively steeper maximum stable slopes as platform width decreases relative to grain diameter, especially when this ratio is less than $\sim 15$ [Barton and Buchberger, 2003]. Lamb et al. [2013] found a similar effect for piles of sand on steep slopes trapped above finite-width barriers. Finite size effects such as these should generally be important in piles of granular material when the addition or removal of a single grain can cause the surface slope to change by more than a few degrees, which corresponds to pile dimensions of $\sim 30$ grain diameters [Liu et al., 1991]. Prancevic et al. [2014] observed that failure of gravel in a steep flume often involved patches $\sim 7$ grain diameters in length and that these small assemblies of grains had a friction angle between the grain-pocket and bulk friction angles.

We hypothesize that there exists a continuous transition from grain-pocket to bulk friction angles as the number of potentially mobile grains increases from one to several tens of grains owing to an increased role of particle force chains [e.g., Cates et al., 1998; Furbish et al., 2008; Sun et al., 2010] in destabilizing the granular mass. Herein we use the terms grain-pocket and bulk friction angle for the end-member cases of single and many grains as defined above, respectively, and we use the term friction angle generically to describe the behavior between and including the end-member cases. We suspect that as force chains become longer, they are increasingly effective at dislodging downhill "keystone" grains with large grain-pocket friction angles, thereby reducing the overall resistance to failure. A material's friction angle therefore may emerge from the underlying probability distribution of individual grain-pocket friction angles.

To test our hypothesis, we carried out physical and numerical experiments in which we isolated the effects of force chains by controlling their orientation and length by placing or simulating linear chains of particles on a rough surface aligned in the steepest downslope direction. This experimental setup deliberately avoids the complications of force chains that could develop in the cross-slope or slope-normal directions within thicker sheets of particles. In the physical experiments we used natural fluvial gravel on a tilting table, so the experiments were subject to the natural variability of grain size and shape, pocket geometry, and contact-point friction but limited in terms of sample size and the length of linear particle chains that could reliably be tested in a laboratory setting. We derived a 1-D probabilistic model that propagates forces downslope based solely on empirical grain-pocket friction angles selected randomly from lognormal distributions, typical of fluvial gravel. The probabilistic model implicitly includes the effects of particle shape and size, grain-pocket geometry, and contact-point friction but does not allow one to unravel the relative role of these effects. Therefore, to further evaluate the role of force chains in grain instability, we used discrete element modeling (DEM), which determines particle motions from first principles, to measure the friction angles of single and short chains of particles on a tilting bed. Discrete element modeling explicitly includes contact-point friction, but we simulated spherical particles only, so these experiments were limited in terms of grain shapes and pocket geometries. Despite different strengths and weaknesses, all three approaches support our hypothesis and reveal a systematic decrease in the friction angle of particles on a tilting bed as the number of particles increases. Below, we first present the experiments and probabilistic model, then the DEM, and finish with a discussion of results, including implications for 3-D particle arrangements. 

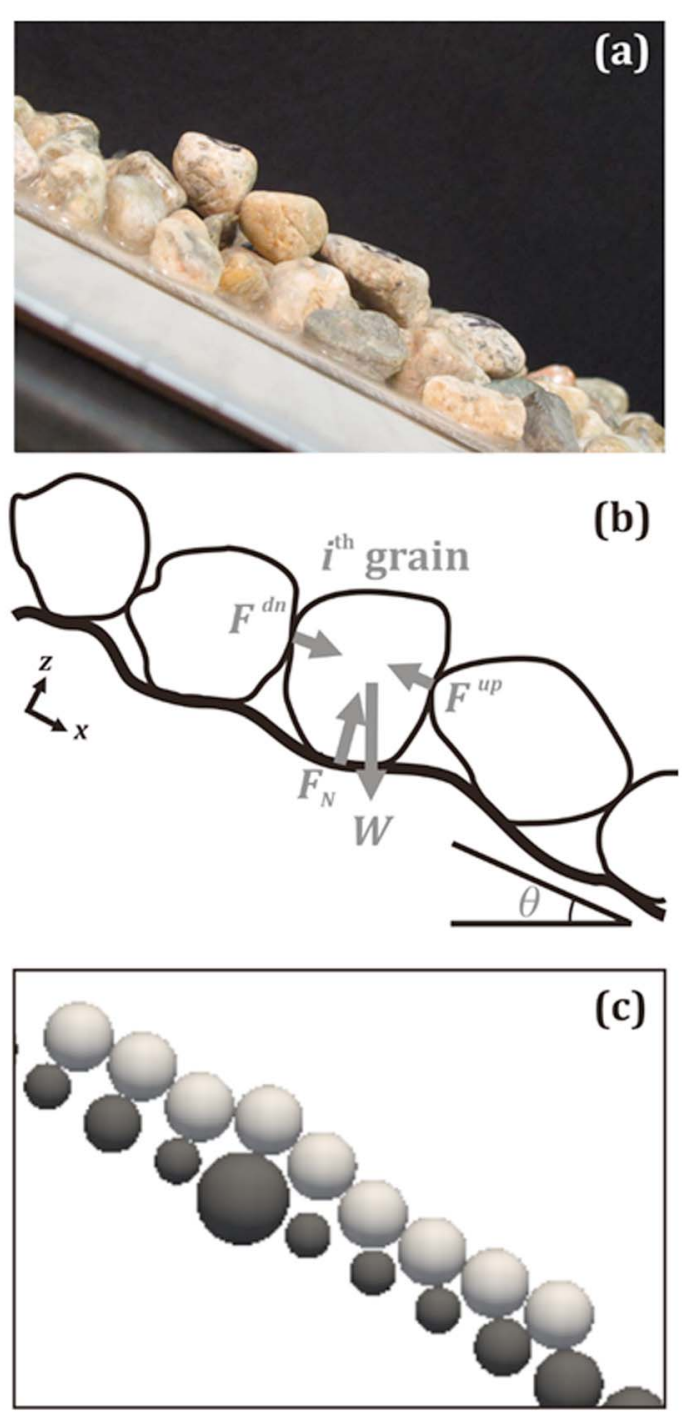

Figure 1. (a) Photograph of a physical experiment in which four gravel particles rest in a line on a tilting bed of gravel epoxied to a board. (b) Forces acting on a grain in the 1-D probabilistic force chain model. The $i^{\text {th }}$ grain potentially feels a downslope force, $F^{\mathrm{dn}}$, resulting from unstable upslope grains, which it transmits to the next grain, equal in magnitude and opposite in direction to $F^{\text {up }}$, only if this force exceeds the grain's frictional resistance (equations (1)-(3)). (c) Example of DEM experiment with a line of $18.7 \mathrm{~mm}$ test spheres (white) resting on a bed of fixed spheres (grey).

\section{Physical Experiments and Probabilistic Model}

\subsection{Experimental Setup}

We carried out a targeted series of physical experiments to measure the friction angle of single grains and small chains of gravel using a tilting table (Figure 1a). The bed of the table consisted of subrounded gravel with a mean intermediate diameter of $15 \mathrm{~mm}$ epoxied to a rigid board. We first measured the grain-pocket friction angles of the gravel by dropping individual grains from a height of several millimeters onto the initially horizontal bed, using different drop locations to sample the entire surface. We then slowly tilted the bed until the grain became unstable and recorded the bed angle, $\theta$, as the grain-pocket friction angle. In addition to single grains, we repeated the procedure with chains of 2 and 4 grains created by dropping the additional gravel particles in a straight line upslope of the lowermost particle so that the grains were just touching. The friction angle for each small assembly of grains was defined as the bed angle, $\theta$, at which any grain or combination of grains became unstable.

\subsection{Model Formulation}

We derived a 1-D probabilistic model for force chains based on the experiments described above (Figure 1b). The model begins by assuming that a single grain rests on a rough, horizontal bed of immobile grains. The grain's resistance to motion (equation (1)) is parameterized by its grain-pocket friction angle, which the model selects randomly from a lognormal distribution [Miller and Byrne, 1966; Kirchner et al., 1990; Buffington et al., 1992]. To simulate a chain $N$ particle in length, the model assumes that additional grains of equal weight lie in a straight line behind the initial grain, with each grain's resistance to motion determined by its randomly chosen grain-pocket friction angle. The initially level surface then tilts in increments of $1^{\circ}$, and at each increment the model calculates the net force in the downslope direction, $F_{i}^{\text {dn }}$, exerted by each grain in the line, where $i$ indexes the grains in the downhill direction (Figure 1a). The first grain exerts a downslope force when the bed angle first exceeds its grain-pocket friction angle:

$$
F_{1}^{\mathrm{dn}}= \begin{cases}W \sin \theta ; & \text { if } \theta>\varphi_{1} \\ 0 ; & \text { if } \theta>\varphi_{1}\end{cases}
$$

where $W$ is the grain's weight, $\theta$ is the angle of the surface from horizontal, and $\varphi_{1}$ is the grain-pocket friction angle. Equation (2) assumes that if the grain becomes unstable, it exerts the full downslope component of its weight on the next particle in the line. Continuing down the line of particles the model recursively calculates

$$
F_{i}^{\mathrm{dn}}= \begin{cases}F_{i-1}^{\mathrm{dn}}+W \sin \theta ; & \text { if } F_{i-1}^{\mathrm{dn}}+W \sin \theta>W \cos \theta \tan \varphi_{i} \\ 0 ; & \text { if } F_{i-1}^{\mathrm{dn}}+W \sin \theta \leq W \cos \theta \tan \varphi_{i}\end{cases}
$$



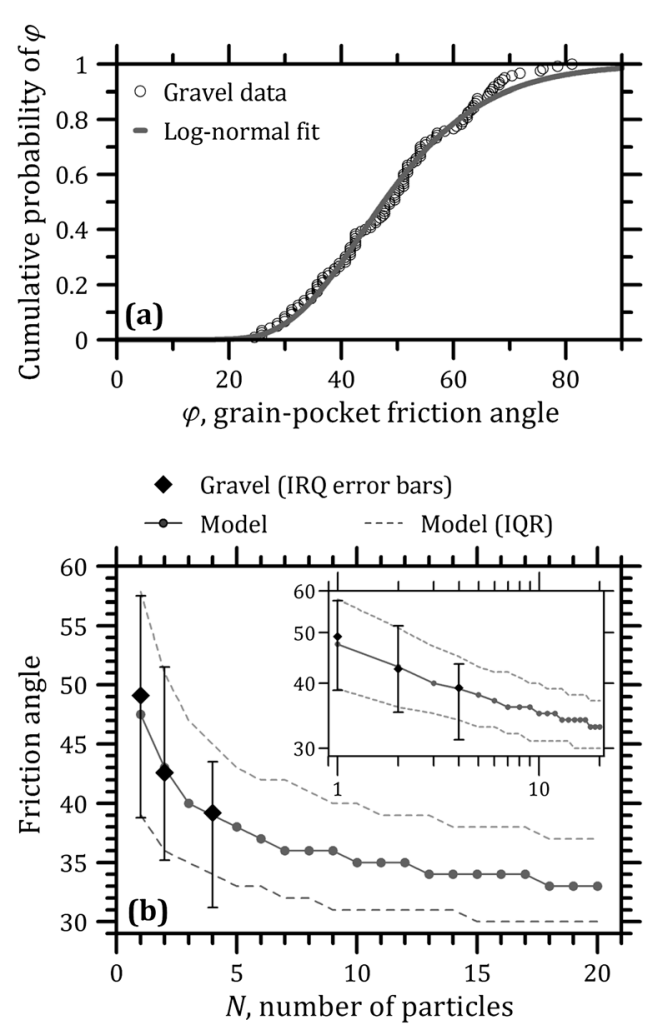
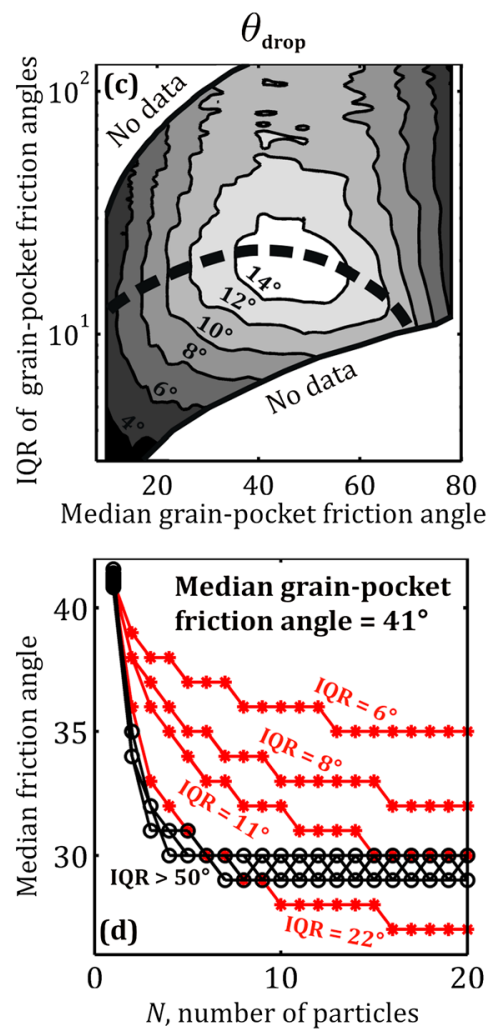

Figure 2. (a) Cumulative probability density function of grain-pocket friction angles for $15 \mathrm{~mm}$ gravel with lognormal fit. (b) Median and IQR of friction angle for different numbers of particles in a line from the gravel experiments (black diamonds with error bars), and the 1-D probabilistic model (gray circles and lines) using grain-pocket friction angles taken randomly from the lognormal fit in Figure 2a. Inset is the same data plotted on logarithmic axes. (c) Magnitude of the drop in friction angle, $\theta_{\mathrm{drop}}$, with increasing $N$ as a function of the median and IQR of the underlying lognormal distribution of grain-pocket friction angles. Dashed line indicates the maximum drop for a given median grain-pocket friction angle. (d) Median friction angle versus $N$ for a lognormal distribution of grain-pocket friction angles with a median of $41^{\circ}$ and IQR ranging from $6^{\circ}$ to $>50^{\circ}$. Black lines with open circles are for distributions with an IQR $>50^{\circ}$, and red lines with asterisks are for IQRs of $22^{\circ}$ or less.

so that if any grain is stable, it prevents forces from being transmitted to the remaining downslope grains. The simulation stops when the lowermost keystone grain becomes dislodged, and the value of $\theta$ defines the friction angle for that assembly of grains. The model does not simulate tilting $>90^{\circ}$, so particles with grain-pocket friction angles $\geq 90^{\circ}$ are always stable. For each value of $N$, we ran 10,000 simulations in order to confidently determine both the central tendency (median) and spread (interquartile range, IQR) of the friction angles.

\subsection{Results}

Like previous work on natural gravel [Miller and Byrne, 1966; Kirchner et al., 1990; Buffington et al., 1992], the distribution of grain-pocket friction angles from the experiments is lognormal with the middle $50 \%$ of the friction angles falling between $39^{\circ}$ and $58^{\circ}$ (Figure $2 a$ ). We used this lognormal fit to randomly generate grain-pocket friction angles in the 1-D probabilistic model. The probabilistic model and physical experiments agree, and both show a decrease in the median friction angle of $\sim 9^{\circ}$ as $N$ increases from 1 to 4 (Figure 2b). For larger values of $N$, the probabilistic model shows a continuing decrease in the median $\theta$, as well as a slight narrowing of the IQR. This rate of decline decreases with increasing $N$ and becomes negligible after $N \sim 20$ (Figure $2 \mathrm{~b}$, inset).

More generally, for any lognormal distribution of grain-pocket friction angles, the 1-D probabilistic model predicts that the size of the drop from grain-pocket to bulk friction angles, denoted $\theta_{\text {drop, }}$ is systematically a function of the distribution's central tendency and spread (Figure $2 \mathrm{c}$ ). We arbitrarily define $\theta_{\text {drop }}$ as the difference between the median friction angle when $N=1$ and the median when $N=20$, since the median 
friction angle either stabilized or continued to decrease only slightly by $N=20$ in all observed numerical experiments. For a given median grain-pocket friction angle, $\theta_{\text {drop }}$ initially increases to a maximum as the distribution's spread becomes larger (Figure 2c). The maximum drop is reached for an IQR of $10^{\circ}$ to $22^{\circ}$ for all distributions of grain-pocket friction angles simulated. As a distribution's spread increases further, the size of the drop gradually decreases until it reaches a constant, and $\theta_{\text {drop }}$ is no longer sensitive to $N$. The maximum drop predicted by the 1-D probabilistic model is $15^{\circ}$ for a distribution with a median of $41^{\circ}$ and an IQR of $22^{\circ}$.

The nature of the transition from high grain-pocket to low-bulk friction angles with increasing $N$ depends on whether the spread of the grain-pocket friction angle distribution is less or greater than the spread corresponding to the maximum drop. Figure $2 \mathrm{~d}$ shows the decrease in median friction angle with increasing $N$ for a lognormal distribution of grain-pocket friction angles with a median of $41^{\circ}$. For the smallest IQRs, $\theta_{\text {drop }}$ is also small, and it increases with the IQR until attaining the maximum $\theta_{\text {drop }}$ at an IQR of $22^{\circ}$. For these small IQRs, the median friction angle decays with increasing $N$ very gradually by $N=20$, consistent with the experimental data (Figure $2 \mathrm{~b}$ ). However, for IQRs of $\sim 50^{\circ}$ or more, the median friction angle reaches a floor by $N \sim 8$ particles beyond which it is no longer sensitive to increases in $N$ or to further increases in the IQR.

\section{Discrete Element Modeling}

\subsection{Methods}

We expanded on the experiments described above by explicitly including contact-point friction and determining particle motion from first principles, using a discrete element model (the granular module of the LAMMPS molecular dynamics code [Plimpton, 1995] available from http://lammps.sandia.gov/). The DEM technique alternates between applying Newton's second law and a force-displacement law to a group of particles in order to determine particle motions and forces at the particle contacts, respectively [e.g., Cundall and Strack, 1979]. During a small increment of time, the linear and angular components of each particle's velocity are determined by integrating Newton's second law with respect to time. These velocities are then integrated to determine the normal and tangential components of the relative particle displacements. The displacements give the normal and tangential forces on each contact point according to the force-displacement law. This law commonly assumes that force is linearly proportional to displacement, but precise modeling of individual particle deformation is generally not necessary to approximate the larger scale mechanical behavior of the granular assembly [Cundall and Strack, 1979]. Sliding at grain-to-grain contacts occurs according to a Coulomb-type friction law if the tangential force exceeds the product of the normal force and the contact-point friction coefficient, $\mu$.

We mimicked the setup of the 1-D probabilistic model and physical experiments in the DEM (Figure 1c). The bed of the tilting surface was $60 \mathrm{~cm}$ long and composed of 30 spheres, spaced $20 \mathrm{~mm}$ on center, and selected randomly from a distribution of spheres with diameters of 9.2, 12.5, 15.6, 18.7, and $25.1 \mathrm{~mm}$ (percent by number of $5 \%, 26 \%, 37 \%, 26 \%$, and $6 \%$, respectively). The 3-D spheres were constrained to lie in a straight line in the downslope direction as assumed for the probabilistic model, and choosing spheres from a distribution of sizes generated a distribution of grain-pocket friction angles while avoiding the computational challenges of complex particle shapes.

For each simulation, a test sphere was placed at a random location on the bed so that it rested in a pocket between two adjacent spheres. Additional test spheres were then placed in the pockets immediately uphill of this sphere so that $N$ spheres lay in a line. Depending on the geometry of the individual pockets, these test spheres were initially in contact with one another or were separated by a distance typically a small fraction of the test sphere diameter (Figure 1c). The bed then slowly tilted until a sphere became dislodged and left the table, at which point the experiment stopped and the current bed angle was defined as the friction angle of that assembly of test spheres. We used test spheres with diameters of 15.6 or $18.7 \mathrm{~mm}$ and contact-point friction coefficients of $\mu=0.1,0.3$, or 0.5 , and we ran 580 simulations for each value of $N$.

\subsection{Results}

Single test spheres failed by rolling, rather than sliding, out of their grain-pockets once the table tilted sufficiently for the test sphere's center of mass to pivot forward from its lowermost contact point with the bed. Thus, the distributions of grain-pocket friction angles are independent of $\mu$ because contact-point friction only affects the threshold for grain-to-grain slip. The distributions of friction angles are lognormal 

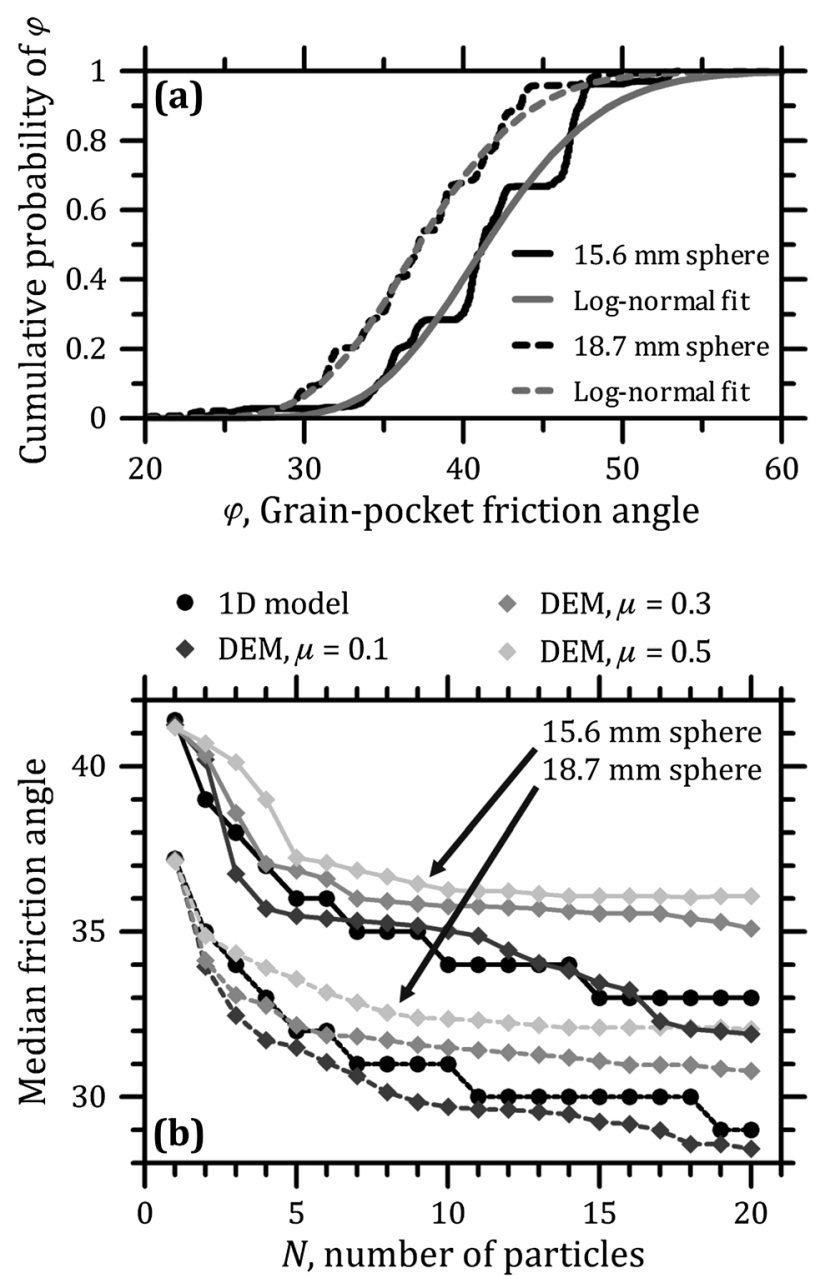

Figure 3. (a) Cumulative density functions of grain-pocket friction angles from the DEM with test spheres of 15.6 and $18.7 \mathrm{~mm}$. (b) Median friction angle versus the number of particles in the line for the $15.6 \mathrm{~mm}$ test spheres (solid lines) and $18.7 \mathrm{~mm}$ test spheres (dashed lines). DEM results are in shades of gray for different values of the contact-point friction coefficient, and 1-D probabilistic model results are in black. with medians and IQRs of $41^{\circ}$ and $10^{\circ}$, and $37^{\circ}$ and $8^{\circ}$, for the 15.6 and $18.7 \mathrm{~mm}$ test spheres, respectively (Figure $3 \mathrm{a}$ ). Grain-pocket friction angles for the $18.7 \mathrm{~mm}$ test sphere are smaller than those of the $15.6 \mathrm{~mm}$ test sphere, all else being equal, because the larger sphere protrudes more from the bed.

Failures involving more than one test sphere occurred by a combination of rolling and sliding at grain contacts, so contact-point friction had an effect on the resulting friction angles (Figure $3 \mathrm{~b}$ ). To dislodge the lowermost keystone particle while the grains remained in contact, sliding occurred at the contact points between adjacent test grains, or at points where the test grains contacted the bed, or both. For a given value of $\mu$, the median friction angle is highest for single particles and decreases at least several degrees as the number of particles increases, consistent with the experiments and 1-D probabilistic model (Figure $3 b$ ). The spheres with the highest $\mu$ have the smallest $\theta_{\text {drop }}$ of $5^{\circ}$, while the spheres with the lowest $\mu$ have the largest drop of $9^{\circ}$, indicating that higher contact-point friction reduces the effect of force chains because a larger portion of a particle's downslope weight is balanced by friction at particle-particle and particle-bed contacts.

To compare the DEM results to the 1-D probabilistic model and experimental data, we used the lognormal fits to the distributions of grain-pocket friction angles (Figure 3a) to run simulations as described

in section 2.2. Results of the probabilistic model most closely track those of the DEM with $\mu=0.1$ (Figure 3b). This suggests that low contact-point friction allows particles to transmit a large proportion of their weight to downslope particles, consistent with full downslope weight transfer as assumed in the probabilistic model (equation (2)). The probabilistic model also matches the experimental data well (section 2.3 and Figure $2 \mathrm{~b}$ ), implying that the contact-point friction of natural gravel is small relative to its grain-pocket and bulk friction angles and that grains are able to transfer a large proportion of their weight downslope.

\section{Discussion and Conclusions}

The friction angle of linear chains of sediment grains in our 1-D and 2-D experiments decreases by a few up to $15^{\circ}$ as more grains are present, and the magnitude of the drop is similar to the difference between grain-pocket and bulk friction angles measured in natural, 3-D materials. We suggest that this agreement results from the competing effects of different force chain orientations in 3-D grain arrangements, rendering the net effect similar to 1-D and 2-D arrangements. For example, in a sheet of grains with a thickness of one grain diameter, force chains can develop in the cross-slope direction so that grains diagonally upslope of a keystone grain play a role in dislodging that keystone grain. However, assuming the sheet of grains is isotropic, these diagonal force chains could just as likely divert force away from the keystone. Similarly, for a 
thick sheet of grains, force chains can develop in the slope-normal direction and contribute either to stabilizing grains at the bottom of the sheet by increasing the normal stress if directed into the slope or to dislodging grains near the surface if directed out of the slope.

Our results bridge the gap in scale between constitutive laws, such as equation (1), applied to granular materials at the macroscale and detailed treatments of grain contact mechanics applied at the microscale [e.g., Andrade and Tu, 2009; Sun et al., 2010]. One way of accounting for force chains in macroscale constitutive laws is to adjust the empirical friction angle according to numerical or physical experiments as described here. For a granular material and substrate with a wide range of grain-pocket friction angles, the adjustment becomes important when $N<8$, while for more uniform distributions it becomes important when $N<20$ or more (Figure 3). These length scales are broadly consistent with others' experimental data involving grain sizes that are large compared to the testing apparatus [Chandler, 1973; Kirkby and Statham, 1975; Carson, 1977; Barton and Buchberger, 2003; Lamb et al., 2013] and with theoretical considerations of finite size effects [Liu et al., 1991]. Furthermore, force chains in dense granular materials are generally shorter than these length scales (e.g., Sun et al. [2010] found that $99 \%$ of force chains are less than 20 particles long) so that friction angles become independent of the number of grains for scales longer than the longest force chains.

The observation that longer force chains more effectively dislodge downslope particles suggests that force chains may control the sizes of small mass failures in granular materials. On average, a short force chain triggering a small failure requires a steeper slope than a long force chain triggering a large failure, rendering the smallest failures relatively uncommon. On the other hand, very large failures at low slopes are relatively uncommon because force chains are generally not long enough to dislodge relatively stable keystone particles. Onda and Matsukura [1997] observed similar failure processes driven by force chains in tilting box experiments involving avalanches of rods. In those experiments, a keystone rod would support the weight of a short chain of uphill rods until the box tilted enough for the chain to dislodge the keystone rod, initiating an avalanche. The bed angle at which the rods failed was distinct from the contact-point friction coefficient, consistent with our results (Figure 3b).

It is increasingly recognized that grain-grain interactions in addition to fluid-grain interactions play an important role in creating clusters of stable particles on riverbeds [Frey and Church, 2009, 2011; Estep and Dufek, 2012]. Our results imply that grain-grain interactions play a role in creating stable clusters because particularly stable grains are often able to support long chains of less stable grains in the upslope direction [Church and Zimmermann, 2007]. Movement of fluid over a grain cluster would likely inhibit the formation of very long chains by exerting an additional stress in the downslope direction to more easily dislodge the keystone grain. In steep channels, fluvial transport and mass failure can both occur depending on characteristics of the flow as well as on the state of the bed [Kean et al., 2013; Prancevic et al., 2014]. Kean et al. [2013] showed that during large flows with high sediment supply, coarse grains can temporarily accumulate on level channel reaches until enough stress builds to overcome the grains' frictional resistance, resulting in debris-flow surges. We suggest that in addition to fluid-grain interactions, force chains acting at grain-grain contacts may control instability by setting the friction angle for the accumulating debris. Initial motion of bed material in steep channels is also often by mass failure, and recent flume experiments show that the friction angle that best predicts failure is associated with force chains $\sim 7$ particles long, rather than either the grain-pocket or the bulk friction angle [Prancevic et al., 2014].

Clusters of small numbers of grains abound on Earth's surface, and we expect the effect of force chains on

Acknowledgments

We thank two anonymous reviewers for constructive feedback that improved the scope of the study. This work was supported by NSF grant EAR-1349115 to M.P.L., and grants from the Terrestrial Hazard and Observation Reporting Center and the Keck Institute for Space Science at Caltech to M.P.L. and J.E.A. Experimental data are available in the supporting information.

The Editor thanks two anonymous reviewers for assistance evaluating this paper. friction angles between grain-pocket and bulk values to be important for wide ranging aeolian, fluvial, and hillslope processes, as well as industrial processes that use granular material. Further quantification of this effect will continue to bridge the gap between constitute approximations, which treat granular material as a continuum, and particle-based laws using first principles, which are computationally expensive.

\section{References}

Andrade, J. E., and X. Tu (2009), Multiscale framework for behavior prediction in granular media, Mech. Mater., 41(6), 652-669, doi:10.1016/j. mechmat.2008.12.005.

Barton, J., and S. Buchberger (2003), Geometrical analysis of sand piles on small platforms, Phys. Rev. E, 68(1), doi:10.1103/ PhysRevE.68.011303.

Behre, C. (1933), Talus behavior above timber in the Rocky Mountains, J. Geol., 41(6), 622-635.

Brayshaw, A. C., L. E. Frostick, and I. Reid (1983), The hydrodynamics of particle clusters and sediment entrainment in coarse alluvial channels, Sedimentology, 30, 137-143, doi:10.1111/j.1365-3091.1983.tb00656.x. 
Buffington, J. M., W. E. Dietrich, and J. W. Kirchner (1992), Friction angle measurements on a naturally formed gravel streambed: Implications for critical boundary shear stress, Water Resour. Res., 28(2), 411-425, doi:10.1029/91WR02529.

Carson, M. A. (1977), Angles of repose, angles of shearing resistance and angles of talus slopes, Earth Surf. Processes Landforms, 2(4), 363-380, doi:10.1002/esp.3290020408.

Cates, M. E., J. P. Wittmer, J. P. Bouchaud, and P. Claudin (1998), Jamming, force chains, and fragile matter, Phys. Rev. Lett., 81(9), 1841-1844, doi:10.1103/PhysRevLett.81.1841.

Chandler, R. J. (1973), The inclination of talus, arctic talus terraces, and other slopes composed of granular materials, J. Geol., 81(1), 1-14.

Church, M., and A. Zimmermann (2007), Form and stability of step-pool channels: Research progress, Water Resour. Res., 43, W03415, doi:10.1029/2006WR005037.

Church, M., M. A. Hassan, and J. F. Wolcott (1998), Stabilizing self-organized structures in gravel-bed stream channels: Field and experimental observations, Water Resour. Res., 34(11), 3169-3179, doi:10.1029/98WR00484.

Cundall, P. A., and O. D. L. Strack (1979), A discrete numerical model for granular assemblies, Geotechnique, 29(1), 47-65, doi:10.1680/ geot.1979.29.1.47.

DiBiase, R. A., and M. P. Lamb (2013), Vegetation and wildfire controls on sediment yield in bedrock landscapes, Geophys. Res. Lett., 40, 1093-1097, doi:10.1002/grl.50277.

DiBiase, R. A., A. M. Heimsath, and K. X. Whipple (2012), Hillslope response to tectonic forcing in threshold landscapes, Earth Surf. Processes Landforms, 37(8), 855-865, doi:10.1002/esp.3205.

Estep, J., and J. Dufek (2012), Substrate effects from force chain dynamics in dense granular flows, J. Geophys. Res., 117, F01028, doi:10.1029/ 2011JF002125.

Frey, P., and M. Church (2009), How river beds move, Science, 325(5947), 1509-1510, doi:10.1126/science.1178516.

Frey, P., and M. Church (2011), Bedload: A granular phenomenon, Earth Surf. Processes Landforms, 36(1), 58-69, doi:10.1002/esp.2103.

Furbish, D. J., M. W. Schmeeckle, and J. J. Roering (2008), Thermal and force-chain effects in an experimental, sloping granular shear flow, Earth Surf. Processes Landforms, 33(13), 2108-2117, doi:10.1002/esp.1655.

Kean, J. W., S. W. McCoy, G. E. Tucker, D. M. Staley, and J. A. Coe (2013), Runoff-generated debris flows: Observations and modeling of surge initiation, magnitude, and frequency, J. Geophys. Res. Earth Surf., 118, 2190-2207, doi:10.1002/jgrf.20148.

Kirchner, J. W., W. E. Dietrich, F. Iseya, and H. Ikeda (1990), The variability of critical shear-stress, friction angle, and grain protrusion in water-worked sediments, Sedimentology, 37(4), 647-672, doi:10.1111/j.1365-3091.1990.tb00627.x.

Kirkby, M. J., and I. Statham (1975), Surface stone movement and scree formation, J. Geol., 83(3), 349-362.

Lamb, M. P., M. Levina, R. A. DiBiase, and B. M. Fuller (2013), Sediment storage by vegetation in steep bedrock landscapes: Theory, experiments, and implications for postfire sediment yield, J. Geophys. Res. Earth Surf., 118, 1-14, doi:10.1002/jgrf.20058.

Lambe, T. W., and R. V. Whitman (1969), Soil Mechanics, John Wiley, New York.

Liu, C.-H., H. Jaeger, and S. Nagel (1991), Finite-size effects in a sandpile, Phys. Rev. A, 43(12), 7091-7092, doi:10.1103/PhysRevA.43.7091.

Miller, R. L., and R. J. Byrne (1966), The angle of repose for a single grain on a fixed rough bed, Sedimentology, 6(4), 303-314, doi:10.1111/ j.1365-3091.1966.tb01897.x.

Onda, Y., and Y. Matsukura (1997), Mechanism for the instability of slopes composed of granular materials, Earth Surf. Processes Landforms, 22, 401-411, doi:10.1002/(SICI)1096-9837(199704)22:4<401::AID-ESP708>3.0.CO;2-C.

Plimpton, S. (1995), Fast parallel algorithms for short-range molecular dynamics, J. Comput. Phys., 117, 1-19, doi:10.1006/jcph.1995.1039.

Prancevic, J. P., M. P. Lamb, and B. M. Fuller (2014), Incipient sediment motion across the river to debris-flow transition, Geology, 42(3), 191-194, doi:10.1130/G34927.1.

Scheingross, J. S., E. W. Winchell, M. P. Lamb, and W. E. Dietrich (2013), Influence of bed patchiness, slope, grain hiding, and form drag on gravel mobilization in very steep streams, J. Geophys. Res. Earth Surf., 118, 982-1001, doi:10.1002/jgrf.20067.

Selby, M. J. (1993), Hillslope Materials and Processes, 2nd ed., Oxford Univ. Press, New York.

Sharp, R. P. (1963), Wind ripples, J. Geol., 71(5), 617-636.

Sun, Q., F. Jin, J. Liu, and G. Zhang (2010), Understanding force chains in dense granular materials, Int. J. Mod. Phys. B, 24(29), 5743-5759, doi:10.1142/S0217979210055780.

Takahashi, T. (2014), Debris Flow: Mechanics, Prediction and Countermeasures, 2nd ed., CRC Press/Balkema, Netherlands.

Wiberg, P. L., and J. D. Smith (1987), Calculations of the critical shear stress for motion of uniform and heterogeneous sediments, Water Resour. Res., 23(8), 1471-1480, doi:10.1029/WR023i008p01471. 